\title{
Percepciones de los estudiantes universitarios sobre las plataformas de formación. Estudio de caso
}

\section{Perceptions of university students of learning platforms. A case study}

\author{
Verónica Marín-Díaz \\ Begoña E. Sampedro-Requena \\ Esther Vega-Gea \\ Universidad de Córdoba, UCO (España)
}

\section{Resumen}

El desarrollo de Internet ha aportado una nueva perspectiva a la educación a distancia, acercando este modelo de enseñanza a todos, de manera universal. Ha supuesto una nueva visión de la enseñanza no presencial, pues ha roto, realmente las barreras espacio y tiempo en lo que a la formación a distancia se refiere. Una herramienta que está jugando un papel cardinal en ello son las plataformas de teleformación (PTM). En el presente estudio presentamos los resultados de una investigación de tipo no experimental y descriptiva realizada con estudiantes de la Facultad de Ciencias de la Educación de la Universidad de Córdoba (España), con el objetivo de determinar la visión que estos tienen, como usuarios, de las plataformas. El principal resultado encontrado ha sido que el alumnado, mientras los hombres se muestran partidarios de su uso en la enseñanza superior, las mujeres, por su parte, emplean más las herramientas que hay en ellas (wikis, foros y chats) que los hombres, para comunicarse dentro de la plataforma. En cuanto a la titulación estudiada los alumnos de Grado de Educación infantil son más proclives a su empleo frente a los de Primaria. Destacar como conclusión inicial, la necesidad por los estudiantes indicada de una mayor vinculación de los docentes con la enseñanza desarrollada a través de este formato, cuestión a su juicio que no se realiza.

Palabras clave: enseñanza a distancia; enseñanza superior; percepciones del alumno; tecnología de la información y la comunicación.

\begin{abstract}
The development of the Internet has added a new perspective to distance education, getting this teaching model closer to everybody in a universal way. This has contributed to a new view of non-face education, as it has really broken the barriers space and time as regards the non-face teaching. A tool playing a decisive role in this is the Learning Management Systems (LMS). In this paper we present the results obtained in a non-experimental and descriptive research carried out with students from the Faculty of Education at the University of Cordoba (Spain), with the principal aim of determining the view that they have, as users,
\end{abstract}


of the platforms. The main result found has been that the students, while men seem to be in favour of using it in higher education, women, mean while, use more the tools found in them (wikis, forum and chats) than men, in order to interact inside the platform. Regarding the grade studied, the pupils of Pre-primary Education are more in favour of using it versus Primary Education. As an initial conclusion, it is worth highlighting the need that the students claim of greater involvement of teachers with the teaching developed through this format, what is not performed from their points of view.

Keywords: distance study; higher education; student's perceptions; information and communication technologies.

Internet es una gran ventana al mundo del aprendizaje. En consecuencia, su horizonte de información se encuentra siempre alejado del individuo, provocando que aquel siempre esté en continuo crecimiento, aportando así al internauta o al sujeto, que quiera asomarse a esa ventana, nuevas experiencias de formación. Vinculada a ella se ha desarrollado todo un sistema de apoyo a la educación a distancia, que no es otro que las plataformas de teleformación. Estas han venido a revolucionar la formación online, acercando la educación a todos los rincones del planeta, universalizando la educación.

En el caso de la educación superior, el desarrollo de los nuevos planes de estudio europeizados, ha conllevado el uso de estas plataformas, si bien las experiencias en torno a ellas son de lo más variado.

En las líneas siguientes hacemos un análisis de las visiones que los estudiantes universitarios tienen de este sistema como apoyo o complemento a la docencia universitaria. Consideramos, que la aportación que estos pueden hacer para la mejora de la educación superior es cardinal dada la fluctuación de la sociedad en general, así como de los planes de estudio que se están presentando en las diferentes universidades en particular.

\section{LA FORMACIÓN ONLINE MEDIADA POR PLATAFORMAS}

Como señalan Marín, Reche y Maldonado (2013, pp. 65-66) en la actualidad hablar de formación en red u online "debe partir de una visión constructivista del proceso en sí mismo, buscando como meta potenciar la adquisición y el desarrollo de competencias que habiliten para una correcta inserción sociolaboral", dado que el desarrollo de los sistemas tecnológicos en los que se imbrican (plataformas de teleformación, en adelante PTM) están en insistente y rápido crecimiento. En consecuencia, el proceso de diseño de este tipo de capacitación, supone que el aprendizaje se encuentre en un continuo reciclaje, lo cual implica que el perfil del usuario de esta se acerque más a una perspectiva abierta, flexible, independiente y colaborativa de la enseñanza. 
Esta instrucción en red conlleva la generación de opiniones encontradas, basadas principalmente en la experiencia del estudiante con el sistema o la PTM (Mirabal, Gómez y González, 2015); no obstante, autores como González (2007), Tello, de Miguel y López (2012), Ku, Tseng y Akarasriwo (2013), Rodríguez y Rivadulla (2015) o Rivadulla (2015), han señalado que posee un número significativo de beneficios, -girando en torno a aspectos tales como el desarrollo de estrategias de formación diversa y en diferentes formatos, la potenciación de distintos tipos de comunicación (sincrónica y asincrónica) entre los individuos, propiciar espacios que fomenten el trabajo colaborativo, cooperativo e interdisciplinar, desarrollar una visión global de las acciones, fácil y rápido acceso a los recursos, estimular las habilidades/ competencias sociales, gran difusión del material creado,...-, que hacen que su empleo deje de ser cuestionado (Erol, 2015).

En la actualidad hay un gran número de PTM, tanto de corte gratuito -Moodlecomo de pago -Blackboard-. Sin embargo, en el caso de las universidades españolas la mayoría se ha inclinado por el empleo de Moodle para desarrollar la formación online de sus títulos. Como señala Rivadulla (2015) esta ofrece, "las herramientas necesarias para la docencia universitaria, tanto presencial como semipresencial o a distancia, y promociona nuevos aprendizajes, facilitando el acceso al material de forma organizada". No obstante, dicha facilidad de acceso y uso, que parece presentar a los usuarios, conlleva no solo un cambio en la cultura formativa sino también en la de aprendizaje, es decir los estudiantes han de saber asumir su nuevo rol dentro de los formatos de enseñanza online (e-learning, blendend learning, ubiquitous learning, etc.) siendo este, principalmente, de un mayor protagonismo (Marín, Reche y Maldonado, 2013; Marín y Cabero, 2015; Sam, 2015).

En consecuencia, si docentes e investigadores la entienden como un elemento facilitador del proceso de enseñanza-aprendizaje (Revuelta y Pérez, 2009; Kopcha, 2010; Danciu y Grosseck, 2011), cabe preguntarse qué papel juega para el estudiante. Teniendo en cuenta, que en estos momentos, el discente universitario se encuentra vinculado al logro de lo que se ha venido a llamar competencia digital para la obtención de su título superior. Ante este panorama el rol del alumnado ante las PTM pasa por una redefinición de visión de la misma, por lo que esta ahora deberá situarse en una perspectiva holística que permita ampliar su formación y generar nuevos valores, habilidades y visiones del mundo educativo y laboral que le rodea.

Este nuevo papel que el alumnado toma, también fruto del desarrollo del Espacio Europeo de Educación Superior, implica conocer cuál es su postura ante el empleo de una herramienta, que de manera tan vertiginosa está cambiando los procesos de enseñanza-aprendizaje y ese es el objeto de este estudio.

\section{MÉTODO}

El objetivo principal que nos planteamos con esta investigación es conocer la percepción que poseen los estudiantes de primero del Grado de Educación Primaria 
y Educación Infantil sobre algunos aspectos de los sistemas de gestión de contenido o plataformas de teleformación, en concreto, Moodle.

¿Por qué centrar el estudio en el alumnado de primer año de estudios superiores? La razón obedece a que es el momento en que se realiza su primera toma de contacto con la PTM, no encontrándose "contaminado" por las opiniones y/o experiencias que los docentes pueden transmitir a lo largo de los cuatro años que duran en la actualidad los estudios de Grado en España, siguiendo de este modo las premisas establecidas por Armijo y McAnally-Salas (2011).

El método de investigación seleccionado es de carácter no experimental y descriptivo (Mateo, 2012), buscando una relación entre las variables dependientes e independientes a posteriori, al posicionarnos en un estudio de tipo ex post-facto.

\section{Población y muestra}

La población, a la que se dirige esta investigación, está compuesta por los estudiantes de $1^{\circ}$ Grado de Educación Primaria e Infantil de la Facultad de Ciencias de la Educación de la Universidad de Córdoba, constituida por un total aproximado de 420 sujetos, siendo la muestra objeto de estudio definida, finalmente, por $\mathrm{N}=281$ participantes, apreciándose una estimación de error muestral de e $=3.4 \%$ y un nivel de confianza del 95\%, con una varianza relativa a nivel de confianza de $\mathrm{z}=1.9599$ y pq (varianza de la población) de 0.25 (dado que tanto a p como a q se les asigna o.5), ya que estos son los mínimos estandarizados permitidos para muestras de población finita (Morales, 2012). Asimismo, el muestreo efectuado ha sido por cuotas (Alaminos, 2006), dado que para la selección de la muestra se han considerado la variable de inicio a las plataformas de teleformación (alumnado de primero) y que se encuentren en los Grados de Educación Primaria e Infantil.

De esta forma, la muestra queda determinada por las siguientes características, el $76.5 \%$ son mujeres y el $23.5 \%$ hombres, estando matriculados en el primer curso el 46.3\% en el Grado de Educación Primaria y el 53.7\% en el Grado de Educación Infantil durante el curso académico 2015/2016. En relación a su edad, el 60.9\% se encuentran en el rango de 18-19 años; el 14.2\% tienen entre 20-21; entre 22-23 años está el 10.7\%; y, más de 24 años el 14.2\%.

Si comparamos la edad con la titulación en la que se encuentran matriculados los participantes (ver gráfico 1), podemos observar que existen diferencias (JiCuadrado=23.232 y p=0.00o). Aunque en ambos grados el porcentaje mayor de estudiantes lo encontramos en mujeres, este se ve alterado al tratarse de un estudio u otro; concretamente en el Grado de Educación Primaria el 36.2\% son hombres frente al 63.8\% de mujeres, mientras que en Educación Infantil es mayor el aumento del porcentaje del sexo femenino (87.9\%) y, solo el $12.1 \%$ son hombres. 
Figura 1. Contingencia edad-titulación

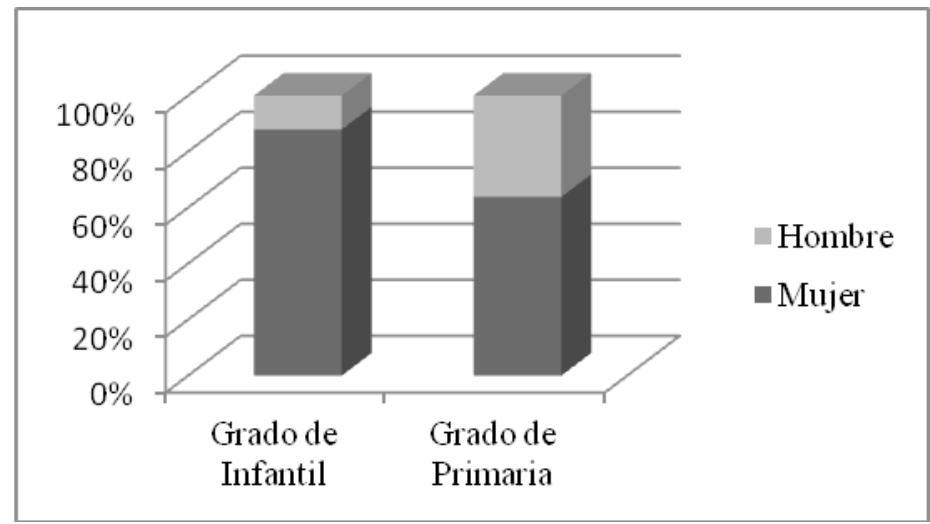

\section{Instrumento}

El instrumento diseñado ad hoc, considera los criterios propuestos por Pérez, García, Gil y Galán (2009) para dotar al mismo de rigor científico; en este sentido, la fiabilidad viene determinada por el análisis de Alfa de Cronbach (o.929), cuyo resultado general indica un grado considerable de consistencia interna. Asimismo, al calcular la correlación entre la puntuación en el ítem y la puntuación en el test (Coeficiente de homogeneidad), los resultados obtenidos reflejan unos coeficientes de a de Cronbach que fluctúan entre 0.927 y 0.930, hecho que induce a no prescindir de ninguno de los ítems, ya que el comportamiento de cada uno de ellos miden un segmento del rasgo que queremos estudiar. Finalmente, buscando la fortaleza en el carácter unidimensional de la prueba (índice de fiabilidad) se ha efectuado el análisis de la capacidad de discriminación de los elementos a través de la prueba estadística t de Student (para muestras independientes, $n . s=0,05$ ) entre las medias de los grupos establecidos, revelando un alto poder de discriminación en todos los ítems $(\mathrm{p}=0,000)$.

Respecto a la validez, se ha efectuado atendiendo al constructo por su nivel de sustancialidad un análisis factorial exploratorio (Hernández, Fernández y Baptista, 2014), para el cual se ha empleado una extracción de elementos principales, atendiendo a aquellos que tenían autovalor mayor que 1, considerando un método de rotación normalización varimax con Kaiser. Previo al mismo se ha efectuado el test de esfericidad de Barllett (ji-cuadrado $=15746.750$ y $\mathrm{p}=0.000$ ) y se ha calculado el índice Kaiser-Meyer-Olkin $(\mathrm{KMO}=0.844)$; el resultado de la extracción de los componentes principales reflejan que existen 8 factores que explican el $70.99 \%$ de la varianza total, lo cual refleja un elevado equilibrio entre los componentes del instrumento. 
En cuanto a su diseño, el instrumento está compuesto de 51 variables, las tres primeras ayudan a describir a la muestra y, las 48 restantes proporcionan información sobre diversos aspectos de las plataformas de teleformación (actitudes generales; experiencia previa y habilidades de uso en herramientas sincrónicas y asincrónicas; creencias didácticas; $\mathrm{y}$, procesos perceptivos y pedagógicos de la PTM), cuya medición se efectúa a través de una escala Likert de cinco opciones de valoración, donde los intervalos corresponden a 1=totalmente en desacuerdo y $5=$ totalmente de acuerdo.

\section{RESULTADOS}

En la siguiente tabla se exponen los resultados de los análisis descriptivos (media y desviación típica) aplicados a los ítems que componen el estudio.

Tabla 1. Medias y desviaciones típicas

\begin{tabular}{|l|c|c|}
\hline ÍTEMS & M. & S. \\
\hline $\begin{array}{l}\text { 1. } \\
\text { en mi medio ambiente }\end{array}$ & 3.32 & 1.098 \\
\hline 2. Prefiero participar en asignaturas semipresenciales & 3.08 & 1.115 \\
\hline $\begin{array}{l}\text { 3. Para aprender en teleformación prefiero realizar cursos cortos (no más } \\
\text { de tres meses) }\end{array}$ & 3.35 & 1.195 \\
\hline 4. Manejo el chat para comunicarme con mis amigos & 3.98 & 1.319 \\
\hline 5. Manejo el chat para comunicarme con mis compañeros de universidad & 3.82 & 1.292 \\
\hline 6. Manejo el chat para comunicarme con el profesorado & 2.49 & 1.257 \\
\hline 7. Manejo los foros para comunicarme con mis amigos & 2.88 & 1.413 \\
\hline 8. Manejo los foros para comunicarme con mis compañeros de universidad & 3.01 & 1.339 \\
\hline 9. Manejo los foros para comunicarme con el profesorado & 2.82 & 1.368 \\
\hline 10. Manejo el correo electrónico para comunicarme con mis amigos & 3.75 & 1.176 \\
\hline $\begin{array}{l}\text { 11. Manejo el correo electrónico para comunicarme con mis compañeros de } \\
\text { universidad }\end{array}$ & 3.74 & 1.195 \\
\hline 12. Manejo el correo electrónico para comunicarme con el profesorado & 3.52 & 1.285 \\
\hline 13. Me conecto a las asignaturas presentes en la PTM en la facultad & 3.48 & 1.444 \\
\hline 14. Me conecto a las asignaturas presentes en la PTM en mi casa & 3.86 & 1.350 \\
\hline 15. Participo en los chats que se convocan en la asignatura & 3.05 & 1.225 \\
\hline 16. Participo en los foros de la asignatura con bastante frecuencia & 2.79 & 1.187 \\
\hline $\begin{array}{l}\text { 17. Respondo a preguntas o iniciativas que algún compañero/a haya enviado } \\
\text { al foro }\end{array}$ & 2.78 & 1.253 \\
\hline 18. La accesibilidad a la PTM es rápida & 3.76 & 1.078 \\
\hline 19. La PTM es fácil de usar ya que su navegación es amigable & 3.74 & 1.099 \\
\hline
\end{tabular}




\begin{tabular}{|c|c|c|}
\hline ÍTEMS & M. & S. \\
\hline $\begin{array}{l}\text { 20. Es fácil realizar actividades individuales y colaborativas a través de la } \\
\text { PTM }\end{array}$ & 3.52 & 1.183 \\
\hline 21. Es sencillo conocer mi progreso y mis evaluaciones a través de la PTM & 3.71 & 1.213 \\
\hline 22. La interfaz de la PTM es dinámica & 3.56 & 1.308 \\
\hline 23. La interfaz de la PTM es didáctica & 3.67 & 1.285 \\
\hline 24. La interfaz de la PTM es motivante & $3 \cdot 37$ & 1.386 \\
\hline 25. La PTM es una herramienta con la cual aprendo de forma más dinámica & 3.55 & 1.319 \\
\hline 26. La PTM es una herramienta con la cual aprendo de forma más sencilla & $3 \cdot 38$ & 1.121 \\
\hline $\begin{array}{l}\text { 27. La PTM me permite relacionarme con personas de otros centros } \\
\text { educativos }\end{array}$ & 2.58 & 1.337 \\
\hline 28. La PTM hace la sesión clase más relajada & 2.99 & 1.283 \\
\hline 29. La PTM hace más flexible mi aprendizaje & $3 \cdot 30$ & 1.264 \\
\hline $\begin{array}{l}\text { 30. En la PTM es fácil identificar dónde consultar el programa de la } \\
\text { asignatura }\end{array}$ & 3.88 & 1.253 \\
\hline $\begin{array}{l}\text { 31. La estructura organizativa de la asignatura se muestra de forma clara } \\
\text { dentro de la PTM: competencias }\end{array}$ & 3.43 & 1.302 \\
\hline $\begin{array}{l}\text { 32. La estructura organizativa de la asignatura se muestra de forma clara } \\
\text { dentro de la PTM: objetivos }\end{array}$ & 3.59 & 1.165 \\
\hline $\begin{array}{l}\text { 33. La estructura organizativa de la asignatura se muestra de forma clara } \\
\text { dentro de la PTM: contenidos }\end{array}$ & 3.78 & 1.171 \\
\hline $\begin{array}{l}\text { 34. La estructura organizativa de la asignatura se muestra de forma clara } \\
\text { dentro de la PTM: metodología }\end{array}$ & 3.60 & 1.167 \\
\hline $\begin{array}{l}\text { 35. La estructura organizativa de la asignatura se muestra de forma clara } \\
\text { dentro de la PTM: evaluación }\end{array}$ & 3.63 & 1.215 \\
\hline $\begin{array}{l}\text { 36. Se accede de forma fácil a la estructura organizativa de la asignatura } \\
\text { dentro de la PTM }\end{array}$ & $3 \cdot 73$ & 1.139 \\
\hline $\begin{array}{l}\text { 37. Prefiero aprender en asignaturas organizativas en temas secuenciados a } \\
\text { través de la PTM }\end{array}$ & $3 \cdot 32$ & 1.263 \\
\hline $\begin{array}{l}\text { 38. Las actividades que se manejan a través de la PTM me incitan a } \\
\text { involucrarme en mi aprendizaje }\end{array}$ & $3 \cdot 33$ & 1.246 \\
\hline $\begin{array}{l}\text { 39. Prefiero que se planteen problemas y trabajar sobre ellos sin un esquema } \\
\text { fijo de contenidos a través de la PTM }\end{array}$ & 2.97 & 1.255 \\
\hline $\begin{array}{l}\text { 40. Considero importante que los docentes dediquen un tiempo a orientarme } \\
\text { sobre la asignatura en la misma PTM }\end{array}$ & $3 \cdot 71$ & 1.207 \\
\hline $\begin{array}{l}\text { 41. Los profesores/as me proporcionan una respuesta rápida a mis dudas a } \\
\text { través de la PTM }\end{array}$ & 3.41 & 1.239 \\
\hline $\begin{array}{l}\text { 42. Los profesores/as me proporcionan una respuesta adecuada a mis dudas } \\
\text { a través de la PTM }\end{array}$ & 3.47 & 1.242 \\
\hline $\begin{array}{l}\text { 43. Los profesores, a través de la PTM, me sugieren estrategias adicionales } \\
\text { para que logre realizar de una mejor manera mis actividades }\end{array}$ & 3.16 & 1.322 \\
\hline
\end{tabular}




\begin{tabular}{|l|c|c|}
\hline ÍTEMS & M. & S. \\
\hline 44. La PTM facilita el aprendizaje activo & 3.50 & 1.199 \\
\hline 45. La PTM supone una mayor autonomía en el proceso de aprendizaje & 3.63 & 1.179 \\
\hline 46. La PTM promueve la habilidad para investigar & 3.53 & 1.177 \\
\hline 47. La PTM permite aprender desde cualquier lugar & 3.83 & 1.181 \\
\hline 48. La PTM permite aprender en cualquier momento & 3.83 & $\mathbf{1 . 1 8 5}$ \\
\hline
\end{tabular}

Nota: $\mathrm{N}=281$ sujetos; PTM= Plataformas de Teleformación.

El alumnado participante indica que, según esta tabla, es fácil de identificar dónde consultar el programa de la asignatura $(\bar{x}=3.88)$, de conectarse a las asignaturas presentes desde casa $(\bar{x}=3.86)$, permiten aprender desde cualquier lugar y momento (ambos $\bar{x}=3.83$ ); muestran claramente los contenidos de la asignatura relativo a la estructura organizativa $(\bar{x}=3.78)$ y se accede fácilmente a esta distribución $(\bar{x}=3.73)$; la accesibilidad es rápida $(\bar{x}=3.76)$; la facilidad en su uso es debido a la navegación amigable $(\bar{x}=3.74)$; consideran importante que los docentes dediquen tiempo a orientar sobre la asignatura en la misma plataforma de teleformación $(\bar{x}=3.71)$.

Por el contrario, están menos de acuerdo con las premisas de usar el chat para comunicarse con el profesorado $(\bar{x}=2.49) \mathrm{y}$, con la idea de que las plataformas de teleformación permiten relacionarse con personas de otros centros educativos $(\bar{x}=2.58)$.

$\mathrm{Al}$ realizar la prueba inferencial t-student (n.s=0.05) para contrastar si existen $o$ no diferencias estadísticamente significativas en función del sexo de la muestra, los resultados muestran que la misma existe en algunos ítems.

Los hombres están más de acuerdo que las mujeres en preferir participar en asignaturas semipresenciales $(\mathrm{t}=-2.267 \mathrm{y} \mathrm{p}=0.024$, ítem 2$)$.

Mientras que las mujeres manejan mejor el chat para comunicarse con sus amigos ( $\mathrm{t}=3.407$ y $\mathrm{p}=0.001$, ítem 4$)$; para comunicarse con sus compañeros de universidad ( $\mathrm{t}=2.666 \mathrm{y} \mathrm{p}=0.009$, ítem5) y comunicarse con el profesorado $(\mathrm{t}=4.718$ y p=0.00o, ítem6) que los hombres. Asimismo, ellas están más de acuerdo en que manejan los foros para comunicarse con sus amigos $(t=2.953$ y $p=0.003$, ítem 7$)$; para comunicarse con sus compañeros de universidad $(\mathrm{t}=5.154 \mathrm{y} \mathrm{p}=0.000$, ítem8) y comunicarse con el profesorado ( $\mathrm{t}=3.464 \mathrm{y} \mathrm{p}=0.001$, ítem9). Igualmente manejan el correo electrónico para comunicarse con sus amigos $(\mathrm{t}=2,951$ y $\mathrm{p}=0,003$, ítem10); comunicarse con sus compañeros de universidad ( $\mathrm{t}=2,508$ y $\mathrm{p}=0,013$, ítem11) y comunicarse con el profesor ( $\mathrm{t}=2,250$ y $\mathrm{p}=0,027$, ítem12) que ellos. 
Figura 2. Comparativa de las medias mujeres-hombres en los ítems del 1 al 12

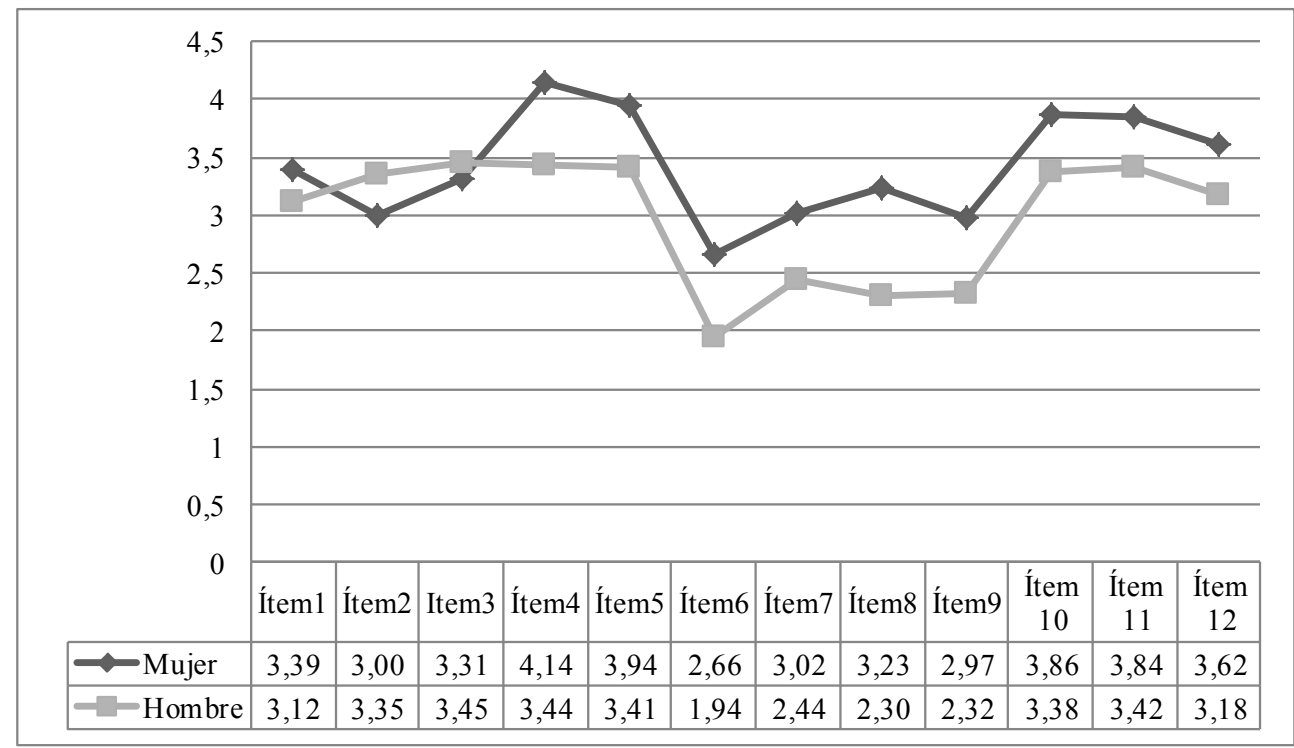

Respecto al uso y la participación, ellas, al contrario que los hombres, señalan estar más de acuerdo en participar en los chats que se convocan en la asignatura $(\mathrm{t}=3.650$ y $\mathrm{p}=0.000$, ítem15).

Las mujeres admiten conectarse a las asignaturas presentes en las plataformas de teleformación en la facultad $(\mathrm{t}=3.839$ y $\mathrm{p}=0.000$, ítem 13$)$ y en su casa $(\mathrm{t}=4.265 \mathrm{y}$ $\mathrm{p}=0.000$, ítem14) más que los hombres.

Las alumnas están más de acuerdo con las siguientes características sobre la interfaz de la PTM: la accesibilidad es más rápida ( $\mathrm{t}=3.325$ y p=0.001, ítem18); la navegación es amigable $(\mathrm{t}=3.508$ y $\mathrm{p}=0.001$, ítem19); su facilidad para realizar actividades individuales y colaborativas $(\mathrm{t}=2.089 \mathrm{y} \mathrm{p}=0.039$, ítem20); la sencillez en el conocimiento del progreso y las evaluaciones $(\mathrm{t}=2.666$ y $\mathrm{p}=0.008$, ítem21); es dinámica $(\mathrm{t}=3.126$ y $\mathrm{p}=0.002, \bar{x}=1$ tem22); es didáctica $(\mathrm{t}=3.310$ y $\mathrm{p}=0.001$, ítem23); y motivante $(\mathrm{t}=3.494$ y $\mathrm{p}=0.001$, ítem24) que los alumnos. 
Figura 3. Comparativa de las medias mujeres-hombres en los ítems 13 al 24

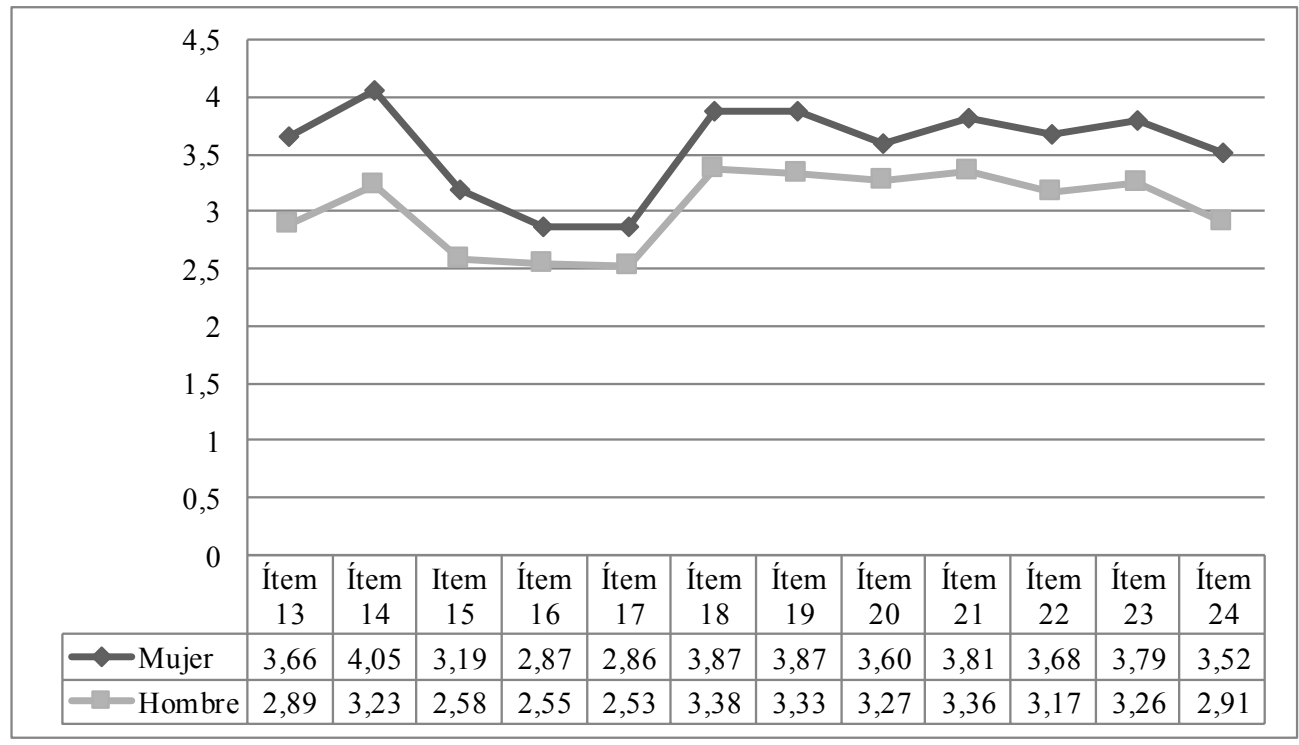

Figura 4. Comparativa de las medias mujeres-hombres en los ítems 25 al 36

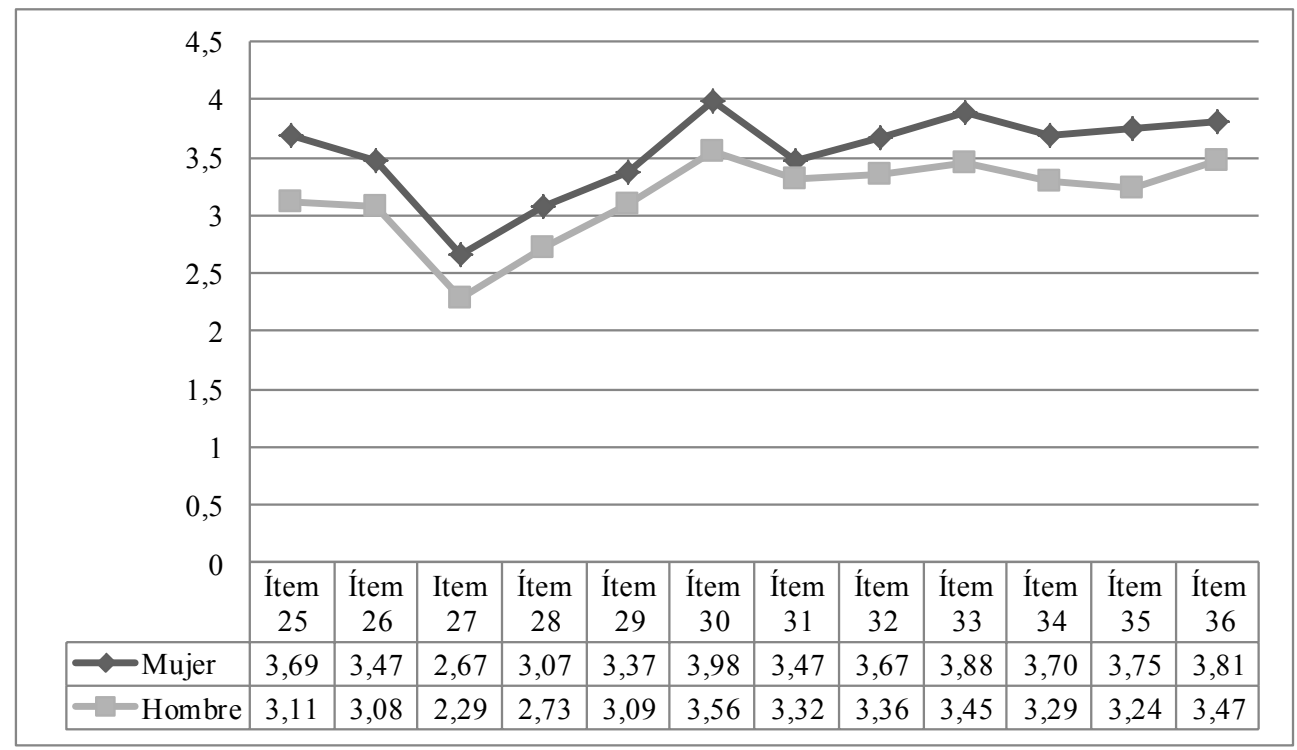


Asimismo (ver figura 4), las mujeres advierten estar más de acuerdo en que la PTM es una herramienta con la que se aprende de forma más dinámica $(\mathrm{t}=3.354 \mathrm{y}$ $\mathrm{p}=0.001$, ítem25); más sencilla $(\mathrm{t}=2.710 \mathrm{y} \mathrm{p}=0.008$, ítem26); permite relacionarse con personas de otros centros educativos $(\mathrm{t}=2.067 \mathrm{y} \mathrm{p}=0.040$, ítem27); $\mathrm{y}$, es fácil identificar donde consultar el programa de la asignatura $(\mathrm{t}=2.585 \mathrm{y} \mathrm{p}=\mathbf{0 . 0 1 1}$, ítem30), que los hombres.

En relación, a la claridad en la que se muestra la estructura organizativa de la asignatura en la PTM, las alumnas están más de acuerdo que los alumnos en que ocurre con los contenidos ( $\mathrm{t}=\mathbf{2 . 6 3 3}$ y p=0.009, ítem33); la metodología $(\mathrm{t}=2.745$ y $\mathrm{p}=0.007$, ítem34); la evaluación ( $\mathrm{t}=3.272$ y p=0.001, ítem35); además, consideran que se accede de forma fácil $(\mathrm{t}=2.132$ y $\mathrm{p}=0.034$, ítem36).

De igual forma, prefieren aprender en asignaturas organizadas en temas secuenciados a través de la PTM $(\mathrm{t}=2.119$ y $\mathrm{p}=0.035$, ítem37); y que las actividades que se manejan a través de las plataformas de teleformación incitan a involucrarse en el propio aprendizaje ( $\mathrm{t}=3.909$ y $\mathrm{p}=0.000$, ítem38) las alumnas más que los alumnos (figura 5).

Por último, las mujeres señalan estar más de acuerdo con la idea de que las PTM suponen una mayor autonomía en el proceso de aprendizaje $(\mathrm{t}=\mathbf{2 . 3 8 4} \mathrm{y} \mathrm{p}=\mathbf{0 . 0 1 8}$, ítem45); permiten aprender desde cualquier lugar ( $\mathrm{t}=2.384 \mathrm{y} \mathrm{p}=0.018$, ítem47); $\mathrm{y}$, permiten aprender en cualquier momento $(\mathrm{t}=2.513$ y $\mathrm{p}=0.013$, ítem48) que los hombres.

Figura 5. Comparativa de las medias mujeres-hombres en los ítems 37 al 48.

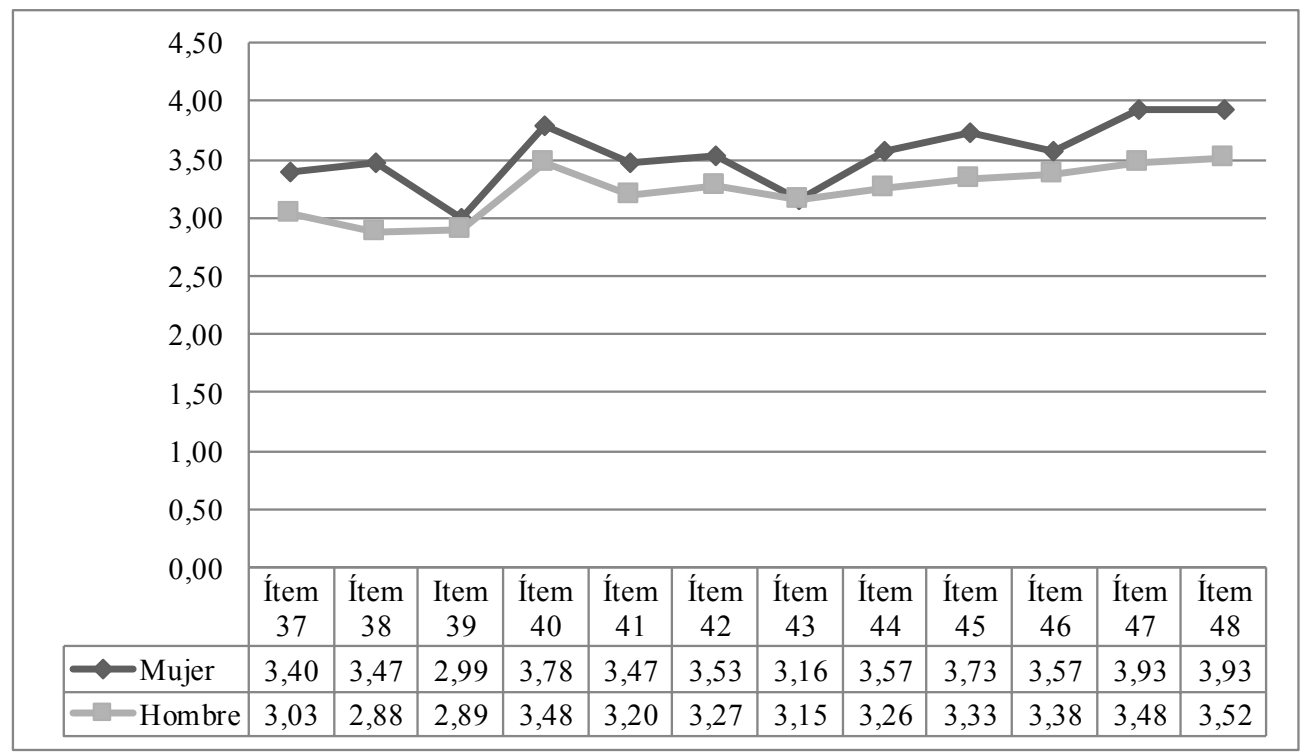


Considerando la edad de la muestra, hemos realizado un análisis de ANOVA (n.s.=0.05), de la que se obtiene que existe un efecto estadísticamente significativo entre algunos ítems y la edad atendiendo a la prueba post hoc de Bonferroni. En este sentido los estudiantes de entre 18-19 años indican manejar el chat para comunicarse con sus amigos $\mathrm{F}(3.277)=2.888, \mathrm{p}=0.036, \mathrm{~h}^{2}=0.030$; con respecto a los de 24 o más t $(277)=2.94$ y p=0.021, en cambio el resto de comparativas de la edad no reflejan resultados estadísticamente significativos. Asimismo, el alumnado más joven señalan mayor manejo del chat para comunicarse con sus compañeros de universidad $\mathrm{F}(3.277)=3.365, \mathrm{p}=0.019, \mathrm{~h}^{2}=0.035$; con respecto al grupo de mayor edad t (277) $=3.09$ y $\mathrm{p}=0.013$, que es el único que expresa diferencias significativas.

Los estudiantes de 22-23 años prefieren aprender en asignaturas organizativas en temas secuenciados a través de la PTM, F (3.277) $=2.990, \mathrm{p}=0.031, \mathrm{~h}^{2}=0.031$, con respecto a los de 18-19 años t $(277)=2.77$ y p=0.036; en cambio el resto de comparativas de la edad no reflejan resultados estadísticamente significativos.

Finalmente, al comparar las medias en función de la titulación en la que se encuentran matriculados los estudiantes participantes mediante el análisis inferencial t-student (n.s=0.05), los resultados arrojados muestran diferencias estadísticamente significativas en algunos ítems.

Tabla 2. T-student en función de la titulación

\begin{tabular}{|c|c|c|c|c|c|}
\hline ÍTEMS & GRADO & $\mathrm{N}$ & Media & s & Тур \\
\hline \multirow{2}{*}{$\begin{array}{l}\text { 1. Considero necesaria la } \\
\text { teleformación por la } \\
\text { escasez de oferta formativa } \\
\text { en mi medio ambiente }\end{array}$} & E. INFANTIL & 151 & 3,45 & 1,094 & \multirow[b]{2}{*}{$\begin{array}{l}\mathrm{T}=2,094 \text { y } \mathrm{p}=0,037 \\
\text { favorable a infantil }\end{array}$} \\
\hline & E. PRIMARIA & 130 & 3,18 & 1,089 & \\
\hline \multirow{2}{*}{$\begin{array}{l}\text { 2. Prefiero participar } \\
\text { en asignaturas } \\
\text { semipresenciales }\end{array}$} & E. INFANTIL & 151 & 3,07 & 1,159 & \multirow{2}{*}{$\begin{array}{l}\mathrm{T}=-0,195 \mathrm{yp}=0,845 \\
\text { (no significativa) }\end{array}$} \\
\hline & E. PRIMARIA & 130 & 3,09 & 1,067 & \\
\hline \multirow{2}{*}{$\begin{array}{l}\text { 3. Para aprender en } \\
\text { teleformación prefiero } \\
\text { realizar cursos cortos (no } \\
\text { más de tres meses) }\end{array}$} & E. INFANTIL & 151 & 3,32 & 1,252 & \multirow[b]{2}{*}{$\begin{array}{l}\mathrm{T}=-0,312 \text { y } \mathrm{p}=0,755 \\
\text { (no significativa) }\end{array}$} \\
\hline & E. PRIMARIA & 130 & 3,37 & 1,128 & \\
\hline \multirow{2}{*}{$\begin{array}{l}\text { 4. Manejo el chat para } \\
\text { comunicarme con mis } \\
\text { amigos }\end{array}$} & E. INFANTIL & 151 & 4,21 & 1,225 & \multirow{2}{*}{$\begin{array}{l}\mathrm{T}=3,274 \text { y } \mathrm{p}=0,001 \\
\text { favorable a infantil }\end{array}$} \\
\hline & E. PRIMARIA & 130 & 3,70 & 1,373 & \\
\hline \multirow{2}{*}{$\begin{array}{l}\text { 5. Manejo el chat para } \\
\text { comunicarme con } \\
\text { mis compañeros de } \\
\text { universidad }\end{array}$} & E. INFANTIL & 151 & 4,00 & 1,260 & \multirow[b]{2}{*}{$\begin{array}{l}\mathrm{T}=2,562 \text { y } \mathrm{p}=0,011 \\
\text { favorable a infantil }\end{array}$} \\
\hline & E. PRIMARIA & 130 & 3,61 & 1,303 & \\
\hline \multirow{2}{*}{$\begin{array}{l}\text { 6. Manejo el chat para } \\
\text { comunicarme con el } \\
\text { profesorado }\end{array}$} & E. INFANTIL & 151 & 2,64 & 1,240 & \multirow{2}{*}{$\begin{array}{l}\mathrm{T}=2,189 \text { y } \mathrm{p}=0,029 \\
\text { favorable a infantil }\end{array}$} \\
\hline & E. PRIMARIA & 130 & 2,32 & 1,258 & \\
\hline
\end{tabular}




\begin{tabular}{|c|c|c|c|c|c|}
\hline ÍTEMS & GRADO & $\mathrm{N}$ & Media & $\mathrm{s}$ & Тур \\
\hline \multirow{2}{*}{$\begin{array}{l}\text { 7. Manejo los foros para } \\
\text { comunicarme con mis } \\
\text { amigos }\end{array}$} & E. INFANTIL & 151 & 3,04 & 1,487 & \multirow{2}{*}{$\begin{array}{l}\mathrm{T}=2,040 \text { y } \mathrm{p}=0,042 \\
\text { favorable a infantil }\end{array}$} \\
\hline & E. PRIMARIA & 130 & 2,70 & 1,304 & \\
\hline \multirow{2}{*}{$\begin{array}{l}\text { 8. Manejo los foros para } \\
\text { comunicarme con } \\
\text { mis compañeros de } \\
\text { universidad }\end{array}$} & E. INFANTIL & 151 & 3,09 & 1,437 & \multirow[b]{2}{*}{$\begin{array}{l}\mathrm{T}=0,982 \text { y } \mathrm{p}=0,327 \\
\text { (no significativa) }\end{array}$} \\
\hline & E. PRIMARIA & 130 & 2,93 & 1,215 & \\
\hline \multirow{2}{*}{$\begin{array}{l}\text { 9. Manejo los foros para } \\
\text { comunicarme con el } \\
\text { profesorado }\end{array}$} & E. INFANTIL & 151 & 2,70 & 1,311 & \multirow{2}{*}{$\begin{array}{l}\mathrm{T}=-1,631 \text { y } \mathrm{p}=0,104 \\
\text { (no significativa) }\end{array}$} \\
\hline & E. PRIMARIA & 130 & 2,96 & 1,422 & \\
\hline \multirow{2}{*}{$\begin{array}{l}\text { 10. Manejo el correo } \\
\text { electrónico para } \\
\text { comunicarme con mis } \\
\text { amigos }\end{array}$} & E. INFANTIL & 151 & 3,92 & 1,158 & \multirow[b]{2}{*}{$\begin{array}{l}\mathrm{T}=2,691 \text { y } \mathrm{p}=0,008 \\
\text { favorable a infantil }\end{array}$} \\
\hline & E. PRIMARIA & 130 & 3,55 & 1,169 & \\
\hline \multirow{2}{*}{$\begin{array}{l}\text { 11. Manejo el correo } \\
\text { electrónico para } \\
\text { comunicarme con } \\
\text { mis compañeros de } \\
\text { universidad }\end{array}$} & E. INFANTIL & 151 & 3,90 & 1,142 & \multirow[b]{2}{*}{$\begin{array}{l}\mathrm{T}=2,393 \text { y } \mathrm{p}=0,017 \\
\text { favorable a infantil }\end{array}$} \\
\hline & E. PRIMARIA & 130 & 3,56 & 1,233 & \\
\hline \multirow{2}{*}{$\begin{array}{l}\text { 12. Manejo el correo } \\
\text { electrónico para } \\
\text { comunicarme con el } \\
\text { profesorado }\end{array}$} & E. INFANTIL & 151 & 3,76 & ,204 & \multirow[b]{2}{*}{$\begin{array}{l}\mathrm{T}=3,523 \text { y } \mathrm{p}=0,000 \\
\text { favorable a infantil }\end{array}$} \\
\hline & E. PRIMARIA & 130 & 3,23 & 1,321 & \\
\hline \multirow{2}{*}{$\begin{array}{l}\text { 13. Me conecto a las } \\
\text { asignaturas presentes en la } \\
\text { PTM en la facultad }\end{array}$} & E. INFANTIL & 151 & 3,63 & 1,495 & \multirow{2}{*}{$\begin{array}{l}\mathrm{T}=1,914 \text { y } \mathrm{p}=0,057 \\
\text { (no significativa) }\end{array}$} \\
\hline & E. PRIMARIA & 130 & 3,30 & 1,368 & \\
\hline \multirow{2}{*}{$\begin{array}{l}\text { 14. Me conecto a las } \\
\text { asignaturas presentes en la } \\
\text { PTM en mi casa }\end{array}$} & E. INFANTIL & 151 & 3,94 & 1,420 & \multirow{2}{*}{$\begin{array}{l}\mathrm{T}=1,108 \text { y } \mathrm{p}=0,269 \\
\text { (no significativa) }\end{array}$} \\
\hline & E. PRIMARIA & 130 & 3,76 & 1,262 & \\
\hline \multirow{2}{*}{$\begin{array}{l}\text { 15. Participo en los chats } \\
\text { que se convocan en la } \\
\text { asignatura }\end{array}$} & E. INFANTIL & 151 & 3,00 & 1,337 & \multirow{2}{*}{$\begin{array}{l}\mathrm{T}=-0,697 \mathrm{y} \\
\mathrm{p}=0,486 \text { (no } \\
\text { significativa) }\end{array}$} \\
\hline & E. PRIMARIA & 129 & 3,10 & 1,081 & \\
\hline \multirow{2}{*}{$\begin{array}{l}\text { 16. Participo en los foros de } \\
\text { la asignatura con bastante } \\
\text { frecuencia }\end{array}$} & E. INFANTIL & 151 & 2,72 & 1,212 & \multirow{2}{*}{$\begin{array}{l}\mathrm{T}=-1,038 \mathrm{y} \\
\mathrm{p}=0,300 \text { (no } \\
\text { significativa) }\end{array}$} \\
\hline & E. PRIMARIA & 130 & 2,87 & 1,157 & \\
\hline \multirow{2}{*}{$\begin{array}{l}\text { 17. Respondo a preguntas } \\
\text { o iniciativas que algún } \\
\text { compañero/a haya enviado } \\
\text { al foro }\end{array}$} & E. INFANTIL & 151 & 2,76 & 1,365 & \multirow[b]{2}{*}{$\begin{array}{l}\mathrm{T}=-0,312 \text { y } \mathrm{p}=0,756 \\
\text { (no significativa) }\end{array}$} \\
\hline & E. PRIMARIA & 130 & 2,81 & 1,114 & \\
\hline \multirow{2}{*}{$\begin{array}{l}\text { 18. La accesibilidad a la PTM } \\
\text { es rápida }\end{array}$} & E. INFANTIL & 151 & 3,83 & 1,112 & \multirow{2}{*}{$\begin{array}{l}\mathrm{T}=1,170 \text { y } \mathrm{p}=0,243 \\
\text { (no significativa) }\end{array}$} \\
\hline & E. PRIMARIA & 130 & 3,68 & 1,036 & \\
\hline
\end{tabular}




\begin{tabular}{|c|c|c|c|c|c|}
\hline ITEMS & GRADO & $\mathrm{N}$ & Media & $\mathrm{s}$ & Тур \\
\hline \multirow{2}{*}{$\begin{array}{l}\text { 19. La PTM es fácil de usar } \\
\text { ya que su navegación es } \\
\text { amigable }\end{array}$} & E. INFANTIL & 151 & 3,85 & 1,182 & \multirow{2}{*}{$\begin{array}{l}\mathrm{T}=1,774 \text { y } \mathrm{p}=0,077 \\
\text { (no significativa) }\end{array}$} \\
\hline & E. PRIMARIA & 130 & 3,62 & ,983 & \\
\hline \multirow{2}{*}{$\begin{array}{l}\text { 20. Es fácil realizar } \\
\text { actividades individuales y } \\
\text { colaborativas a través de } \\
\text { la PTM }\end{array}$} & E. INFANTIL & 151 & 3,68 & 1,293 & \multirow[b]{2}{*}{$\begin{array}{l}\mathrm{T}=2,550 \text { y } \mathrm{p}=0,011 \\
\text { favorable a infantil }\end{array}$} \\
\hline & E. PRIMARIA & 130 & 3,33 & 1,015 & \\
\hline \multirow{2}{*}{$\begin{array}{l}\text { 21. Es sencillo conocer } \\
\text { mi progreso y mis } \\
\text { evaluaciones a través de } \\
\text { la PTM }\end{array}$} & E. INFANTIL & 151 & 3,75 & 1,271 & \multirow[b]{2}{*}{$\begin{array}{l}\mathrm{T}=0,597 \text { y } \mathrm{p}=0,551 \\
\text { (no significativa) }\end{array}$} \\
\hline & E. PRIMARIA & 130 & 3,66 & 1,145 & \\
\hline \multirow{2}{*}{$\begin{array}{l}\text { 22. La interfaz de la PTM es } \\
\text { dinámica }\end{array}$} & E. INFANTIL & 151 & 3,66 & 1,400 & \multirow{2}{*}{$\begin{array}{l}\mathrm{T}=1,357 \text { y } \mathrm{p}=0,176 \\
\text { (no significativa) }\end{array}$} \\
\hline & E. PRIMARIA & 130 & 3,45 & 1,188 & \\
\hline \multirow{2}{*}{$\begin{array}{l}\text { 23. La interfaz de la PTM es } \\
\text { didáctica }\end{array}$} & E. INFANTIL & 151 & 3,81 & 1,370 & \multirow{2}{*}{$\begin{array}{l}\mathrm{T}=2,038 \text { y } \mathrm{p}=0,042 \\
\text { favorable a infantil }\end{array}$} \\
\hline & E. PRIMARIA & 130 & 3,50 & 1,163 & \\
\hline \multirow{2}{*}{$\begin{array}{l}\text { 24. La interfaz de la PTM es } \\
\text { motivante }\end{array}$} & E. INFANTIL & 151 & 3,46 & 1,446 & \multirow{2}{*}{$\begin{array}{l}\mathrm{T}=1,086 \text { y } \mathrm{p}=0,278 \\
\text { (no significativa) }\end{array}$} \\
\hline & E. PRIMARIA & 130 & 3,28 & 1,312 & \\
\hline \multirow{2}{*}{$\begin{array}{l}\text { 25. La PTM es una } \\
\text { herramienta con la cual } \\
\text { aprendo de forma más } \\
\text { dinámica }\end{array}$} & E. INFANTIL & 151 & 3,62 & 1,399 & \multirow[b]{2}{*}{$\begin{array}{l}\mathrm{T}=0,889 \text { y } \mathrm{p}=\mathrm{O}, 375 \\
\text { (no significativa) }\end{array}$} \\
\hline & E. PRIMARIA & 130 & 3,48 & 1,221 & \\
\hline \multirow{2}{*}{$\begin{array}{l}\text { 26. La PTM es una } \\
\text { herramienta con la cual } \\
\text { aprendo de forma más } \\
\text { sencilla }\end{array}$} & E. INFANTIL & 151 & 3,38 & 1,210 & \multirow[b]{2}{*}{$\begin{array}{l}\mathrm{T}=0,004 \text { y } \mathrm{p}=0,997 \\
\text { (no significativa) }\end{array}$} \\
\hline & E. PRIMARIA & 130 & 3,38 & 1,014 & \\
\hline \multirow{2}{*}{$\begin{array}{l}\text { 27. La PTM me permite } \\
\text { relacionarme con personas } \\
\text { de otros centros educativos }\end{array}$} & E. INFANTIL & 151 & 2,72 & 1,466 & \multirow{2}{*}{$\begin{array}{l}\mathrm{T}=1,910 \text { y } \mathrm{p}=0,057 \\
\text { (no significativa) }\end{array}$} \\
\hline & E. PRIMARIA & 130 & 2,42 & 1,154 & \\
\hline \multirow{2}{*}{$\begin{array}{l}\text { 28. La PTM hacen la sesión } \\
\text { clase más relajada }\end{array}$} & E. INFANTIL & 151 & 3,07 & 1,447 & \multirow{2}{*}{$\begin{array}{l}\mathrm{T}=1,204 \text { y } \mathrm{p}=0,230 \\
\text { (no significativa) }\end{array}$} \\
\hline & E. PRIMARIA & 130 & 2,89 & 1,058 & \\
\hline \multirow{2}{*}{$\begin{array}{l}\text { 29. La PTM hace más flexible } \\
\text { mi aprendizaje }\end{array}$} & E. INFANTIL & 151 & 3,40 & 1,420 & \multirow{2}{*}{$\begin{array}{l}\mathrm{T}=1,486 \text { y } \mathrm{p}=0,138 \\
\text { (no significativa) }\end{array}$} \\
\hline & E. PRIMARIA & 130 & 3,18 & 1,048 & \\
\hline \multirow{2}{*}{$\begin{array}{l}\text { 30. En la PTM es fácil } \\
\text { identificar dónde consultar } \\
\text { el programa de la } \\
\text { asignatura }\end{array}$} & E. INFANTIL & 151 & 3,93 & 1,292 & \multirow[b]{2}{*}{$\begin{array}{l}\mathrm{T}=0,693 \text { y } \mathrm{p}=0,489 \\
\text { (no significativa) }\end{array}$} \\
\hline & E. PRIMARIA & 130 & 3,82 & 1,210 & \\
\hline \multirow{2}{*}{$\begin{array}{l}\text { 31. La estructura organizativa } \\
\text { de la asignatura se muestra } \\
\text { de forma clara dentro de la } \\
\text { PTM: competencias }\end{array}$} & E. INFANTIL & 151 & 3,62 & 1,464 & \multirow[b]{2}{*}{$\begin{array}{l}\mathrm{T}=2,758 \text { y } \mathrm{p}=0,006 \\
\text { favorable a infantil }\end{array}$} \\
\hline & E. PRIMARIA & 130 & 3,21 & 1,047 & \\
\hline
\end{tabular}




\begin{tabular}{|c|c|c|c|c|c|}
\hline ITEMS & GRADO & $\mathrm{N}$ & Media & S & Т y p \\
\hline \multirow{2}{*}{$\begin{array}{l}\text { 32. La estructura organizativa } \\
\text { de la asignatura se muestra } \\
\text { de forma clara dentro de la } \\
\text { PTM: objetivos }\end{array}$} & E. INFANTIL & 151 & 3,75 & 1,333 & \multirow[b]{2}{*}{$\begin{array}{l}\mathrm{T}=2,478 \text { y } \mathrm{p}=0,014 \\
\text { favorable a infantil }\end{array}$} \\
\hline & E. PRIMARIA & 130 & 3,42 & ,905 & \\
\hline \multirow{2}{*}{$\begin{array}{l}\text { 33. La estructura organizativa } \\
\text { de la asignatura se muestra } \\
\text { de forma clara dentro de la } \\
\text { PTM: contenidos }\end{array}$} & E. INFANTIL & 151 & 3,96 & 1,296 & \multirow[b]{2}{*}{$\begin{array}{l}\mathrm{T}=2,828 \text { y } \mathrm{p}=0,005 \\
\text { favorable a infantil }\end{array}$} \\
\hline & E. PRIMARIA & 130 & 3,58 & ,971 & \\
\hline \multirow{2}{*}{$\begin{array}{l}\text { 34. La estructura organizativa } \\
\text { de la asignatura se muestra } \\
\text { de forma clara dentro de la } \\
\text { PTM: metodología }\end{array}$} & E. INFANTIL & 151 & 3,81 & 1,274 & \multirow[b]{2}{*}{$\begin{array}{l}\mathrm{T}=3,256 \text { y } \mathrm{p}=0,001 \\
\text { favorable a infantil }\end{array}$} \\
\hline & E. PRIMARIA & 130 & 3,37 & ,982 & \\
\hline \multirow{2}{*}{$\begin{array}{l}\text { 35. La estructura organizativa } \\
\text { de la asignatura se muestra } \\
\text { de forma clara dentro de la } \\
\text { PTM: evaluación }\end{array}$} & E. INFANTIL & 151 & 3,83 & 1,319 & \multirow[b]{2}{*}{$\begin{array}{l}\mathrm{T}=3,142 \text { y } \mathrm{p}=0,002 \\
\text { favorable a infantil }\end{array}$} \\
\hline & E. PRIMARIA & 130 & 3,39 & 1,038 & \\
\hline \multirow{2}{*}{$\begin{array}{l}\text { 36. Se accede de forma fácil a } \\
\text { la estructura organizativa } \\
\text { de la asignatura dentro de } \\
\text { la PTM }\end{array}$} & E. INFANTIL & 151 & 3,89 & 1,257 & \multirow[b]{2}{*}{$\begin{array}{l}\mathrm{T}=2,578 \text { y } \mathrm{p}=0,010 \\
\text { favorable a infantil }\end{array}$} \\
\hline & E. PRIMARIA & 130 & 3,55 & ,957 & \\
\hline \multirow{2}{*}{$\begin{array}{l}\text { 37. Prefiero aprender en } \\
\text { asignaturas organizativas } \\
\text { en temas secuenciados a } \\
\text { través de la PTM }\end{array}$} & E. INFANTIL & 151 & 3,50 & 1,356 & \multirow[b]{2}{*}{$\begin{array}{l}\mathrm{T}=2,638 \text { y } \mathrm{p}=0,009 \\
\text { favorable a infantil }\end{array}$} \\
\hline & E. PRIMARIA & 130 & 3,11 & 1,115 & \\
\hline \multirow{2}{*}{$\begin{array}{l}\text { 38. Las actividades que } \\
\text { se manejan a través } \\
\text { de la PTM me incitan } \\
\text { a involucrarme en mi } \\
\text { aprendizaje }\end{array}$} & E. INFANTIL & 151 & 3,54 & 1,355 & \multirow[b]{2}{*}{$\begin{array}{l}\mathrm{T}=3,022 \text { y } \mathrm{p}=0,003 \\
\text { favorable a infantil }\end{array}$} \\
\hline & E. PRIMARIA & 130 & 3,10 & 1,063 & \\
\hline \multirow{2}{*}{$\begin{array}{l}\text { 39. Prefiero que se planteen } \\
\text { problemas y trabajar sobre } \\
\text { ellos sin un esquema fijo } \\
\text { de contenidos a través de } \\
\text { la PTM }\end{array}$} & E. INFANTIL & 151 & 3,09 & 1,361 & \multirow[b]{2}{*}{$\begin{array}{l}\mathrm{T}=1,707 \text { y } \mathrm{p}=0,089 \\
\text { (no significativa) }\end{array}$} \\
\hline & E. PRIMARIA & 130 & 2,83 & 1,108 & \\
\hline \multirow{2}{*}{$\begin{array}{l}\text { 40. Considero importante que } \\
\text { los docentes dediquen un } \\
\text { tiempo a orientarme sobre } \\
\text { la asignatura en la misma } \\
\text { PTM }\end{array}$} & E. INFANTIL & 151 & 3,99 & 1,249 & \multirow[b]{2}{*}{$\begin{array}{l}\mathrm{T}=4,241 \mathrm{y}=0,000 \\
\text { favorable a infantil }\end{array}$} \\
\hline & E. PRIMARIA & 130 & 3,39 & 1,075 & \\
\hline \multirow{2}{*}{$\begin{array}{l}\text { 41. Los profesores/as me } \\
\text { proporcionan una } \\
\text { respuesta rápida a mis } \\
\text { dudas a través de la PTM }\end{array}$} & E. INFANTIL & 151 & 3,64 & 1,318 & \multirow[b]{2}{*}{$\begin{array}{l}\mathrm{T}=3,465 \text { y } \mathrm{p}=0,001 \\
\text { favorable a infantil }\end{array}$} \\
\hline & E. PRIMARIA & 130 & 3,14 & 1,084 & \\
\hline
\end{tabular}




\begin{tabular}{|c|c|c|c|c|c|}
\hline ITEMS & GRADO & $\mathrm{N}$ & Media & s & Тур \\
\hline \multirow{2}{*}{$\begin{array}{l}\text { 42. Los profesores/as me } \\
\text { proporcionan una } \\
\text { respuesta adecuada a mis } \\
\text { dudas a través de la PTM }\end{array}$} & E. INFANTIL & 151 & 3,60 & 1,352 & \multirow[b]{2}{*}{$\begin{array}{l}\mathrm{T}=1,920 \text { y } \mathrm{p}=0,056 \\
\text { (no significativa) }\end{array}$} \\
\hline & E. PRIMARIA & 130 & 3,32 & 1,087 & \\
\hline \multirow{2}{*}{$\begin{array}{l}\text { 43. Los profesores, a través } \\
\text { de la PTM, me sugieren } \\
\text { estrategias adicionales } \\
\text { para que logre realizar de } \\
\text { una mejor manera mis } \\
\text { actividades }\end{array}$} & E. INFANTIL & 151 & 3,18 & 1,391 & \multirow[b]{2}{*}{$\begin{array}{l}\mathrm{T}=0,303 \text { y } \mathrm{p}=0,762 \\
\text { (no significativa) }\end{array}$} \\
\hline & E. PRIMARIA & 130 & 3,13 & 1,241 & \\
\hline \multirow{2}{*}{$\begin{array}{l}\text { 44. La PTM facilita el } \\
\text { aprendizaje activo }\end{array}$} & E. INFANTIL & 151 & 3,69 & 1,282 & \multirow{2}{*}{$\begin{array}{l}\mathrm{T}=2,952 \text { y } \mathrm{p}=0,003 \\
\text { favorable a infantil }\end{array}$} \\
\hline & E. PRIMARIA & 130 & 3,28 & 1,057 & \\
\hline \multirow{2}{*}{$\begin{array}{l}\text { 45. La PTM supone una mayor } \\
\text { autonomía en el proceso } \\
\text { de aprendizaje }\end{array}$} & E. INFANTIL & 151 & 3,88 & 1,211 & \multirow{2}{*}{$\begin{array}{l}\mathrm{T}=3,884 \text { y } \mathrm{p}=0,000 \\
\text { favorable a infantil }\end{array}$} \\
\hline & E. PRIMARIA & 130 & 3,35 & 1,076 & \\
\hline \multirow{2}{*}{$\begin{array}{l}\text { 46. La PTM promueve la } \\
\text { habilidad para investigar }\end{array}$} & E. INFANTIL & 151 & 3,70 & 1,286 & \multirow{2}{*}{$\begin{array}{l}\mathrm{T}=2,663 \text { y } \mathrm{p}=0,008 \\
\text { favorable a infantil }\end{array}$} \\
\hline & E. PRIMARIA & 130 & 3,33 & 1,007 & \\
\hline \multirow{2}{*}{$\begin{array}{l}\text { 47. La PTM permite aprender } \\
\text { desde cualquier lugar }\end{array}$} & E. INFANTIL & 151 & 3,97 & 1,246 & \multirow{2}{*}{$\begin{array}{l}\mathrm{T}=2,176 \text { y } \mathrm{p}=0,030 \\
\text { favorable a infantil }\end{array}$} \\
\hline & E. PRIMARIA & 130 & 3,66 & 1,082 & \\
\hline \multirow{2}{*}{$\begin{array}{l}\text { 48. La PTM permite aprender } \\
\text { en cualquier momento }\end{array}$} & E. INFANTIL & 151 & 4,02 & 1,225 & \multirow{2}{*}{$\begin{array}{l}\mathrm{T}=2,890 \text { y } \mathrm{p}=0,004 \\
\text { favorable a infantil }\end{array}$} \\
\hline & E. PRIMARIA & 130 & 3,62 & 1,102 & \\
\hline
\end{tabular}

Nota: $\mathrm{PTM}=$ Plataformas de Teleformación.

Los alumnos de primero de Educación Infantil consideran necesaria la teleformación por la escasez de oferta formativa en su entorno $(\mathrm{t}=2.094$ y p=0.037, $\bar{x}=3.45$ ), respecto a los de Educación Primaria.

Asimismo, la muestra del grado de Educación Infantil manejan mejor el chat para comunicarse con sus amigos $(\mathrm{t}=3.274 \mathrm{y} \mathrm{p}=0.001, \bar{x}=4.21)$; para comunicarse con sus compañeros de universidad $(\mathrm{t}=2.562$ y $\mathrm{p}=0.011, \bar{x}=4,00)$ y comunicarse con el profesorado ( $\mathrm{t}=2.189$ y p=0.029, $\bar{x}=2.64)$ que los de Educación Primaria. Asimismo, los de infantil están más de acuerdo en que manejan los foros para comunicarse con sus amigos ( $\mathrm{t}=2.040$ y $\mathrm{p}=0.042 . \bar{x}=3.04)$; igualmente manejan el correo electrónico para comunicarse con sus amigos $(\mathrm{t}=2.691 \mathrm{y} \mathrm{p}=0.008, \bar{x}=3.92)$; comunicarse con sus compañeros de universidad $(\mathrm{t}=2.393$ y $\mathrm{p}=0.017, \bar{x}=3.90)$ y comunicarse con el profesorado ( $\mathrm{t}=3.523 \mathrm{y} \mathrm{p}=0.000, \bar{x}=3.76)$ que los de primaria. Los estudiantes del grado de Educación Infantil están más de acuerdo con las siguientes características sobre la interfaz de la PTM: su facilidad para realizar actividades individuales y colaborativas $(\mathrm{t}=2.550$ y $\mathrm{p}=0.011, \bar{x}=3.68) ; \mathrm{y}$ es didáctica $(\mathrm{t}=2.038$ y $\mathrm{p}=0.042$, $\bar{x}=3.81$, que los de Educación Primaria.

En relación, a la claridad en la que se muestra la estructura organizativa de la asignatura en la PTM, el grado de Educación Infantil está más de acuerdo que los 
de Educación Primaria en qué ocurre con las competencias $(\mathrm{t}=2.758$ y $\mathrm{p}=0.006$, $\bar{x}=3.62)$; con los objetivos $(\mathrm{t}=2.478$ y $\mathrm{p}=0.014, \bar{x}=3.75)$; con los contenidos $(\mathrm{t}=2.828$ y $\mathrm{p}=0.001, \bar{x}=3.96)$; la metodología $(\mathrm{t}=3.256$ y $\mathrm{p}=0.001, \bar{x}=3.81)$; , la evaluación $(\mathrm{t}=3.142$ y $\mathrm{p}=0.002, \bar{x}=3.83)$; además, consideran que se accede de forma fácil $(\mathrm{t}=2.578$ y $\mathrm{p}=0.010, \bar{x}=3.89)$; prefieren aprender en asignaturas organizadas en temas secuenciados a través de la PTM $(\mathrm{t}=2.638$ y p=0.009, $\bar{x}=3.50)$; y que las actividades que se manejan a través de las plataformas de teleformación incitan a involucrarse en el propio aprendizaje $(\mathrm{t}=3.022 \mathrm{y} \mathrm{p}=0.003, \bar{x}=3.54)$.

Los estudiantes matriculados en Educación Infantil consideran importante que los docentes dediquen un tiempo a orientar sobre la asignatura $(\mathrm{t}=4.241$ y $\mathrm{p}=0.000$, $\bar{x}=3.99$ ); y, que proporcionen una respuesta rápida a las dudas en la misma PTM $(\mathrm{t}=3.465$ y $\mathrm{p}=0.001, \bar{x}=3.64)$ con respecto al grado de Educación Primaria.

Por último, el grado de Educación Infantil señala estar más de acuerdo con la idea de que las PTM facilitan el aprendizaje activo $(\mathrm{t}=2.952 \mathrm{y} \mathrm{p}=0.003, \bar{x}=3.69)$; suponen una mayor autonomía en el proceso de aprendizaje $(\mathrm{t}=3.884$ y $\mathrm{p}=0.000, \bar{x}=3.88)$; promueven la habilidad para investigar $(\mathrm{t}=2.663 \mathrm{y} \mathrm{p}=0.008, \bar{x}=3.70)$; permiten aprender desde cualquier lugar $(\mathrm{t}=2.176$ y $\mathrm{p}=0.030, \bar{x}=3.97)$; , permiten aprender en cualquier momento $(\mathrm{t}=2.890$ y $\mathrm{p}=0.004, \bar{x}=4.02)$ que los alumnos matriculados en el grado de Educación Primaria.

\section{CONCLUSIONES}

La incorporación de las PTM en la educación superior es una realidad hoy incuestionable (De Pablos, Colás y González, 2011). Fruto de ello encontramos un nuevo sistema de enseñanza que, como ya hemos indicado anteriormente, presenta aspectos positivos (Lai y Savage, 2013) como limitaciones (Castaño, Jenero y Flores, 2012; Wu y Hung, 2013). No obstante, su empleo generalizado conlleva que los estudiantes receptores de este recurso, generen una opinión tanto hacia las herramientas como hacia el propio sistema en sí.

En el caso de los alumnos de la Universidad de Córdoba, participantes en este estudio, se manifiestan abiertos a su empleo, por lo que es positiva su percepción de un desarrollo de su proceso de aprendizaje a través de las PTM, en este caso Moodle (Green, Inam y Denton, 2012; Maz, Barcho, Jimenez y Adamuz, 2012; Felpeto, Rey, Fernández-Vázquez y Garrote, 2015; Juhary, 2014; Srichanyachon, 2014), sin embargo, si atendemos al género, vemos que los hombres son los que más se decantan en su participación en este tipo de formación.

Como hemos podido constatar, el alumnado sabe emplear las herramientas digitales como foros o wikis vinculados a las plataformas (Boza y Conde, 2015), al contrario de lo que sugieren el estudio de Guel, Pintor y Gómez (2016), siendo las mujeres las que más las emplean y las que más participan en los que se desarrollan en la asignatura (Sam, 2015). 
De los posibles usos que se le pueden dar a las PTM, los estudiantes señalan que las utilizan fundamentalmente para consultar el programa de la asignatura (Felpeto et al., 2015; Rodríguez y Rivadulla, 2015; Rivadulla, 2015), siendo las mujeres las que más indican su empleo.

En lo que se refiere a las ventajas de su empleo, consideran que tanto el fácil como el rápido acceso a la información, la claridad con que se muestran los contenidos y la estructura organizativa de la materia (Juhary, 2014; Eskandari y Soleimani, 2016; Wu y Hung, 2013) son aspectos destacables.

Los aspectos positivos que contemplan los estudiantes, en general, la facilidad que brinda para el empleo y potenciación del trabajo autónomo y colaborativo (Emilynova y Voronina, 2014; Rodríguez y Rivadulla, 2015), si bien las mujeres en particular indican que una interfaz amigable (Wu y Hung, 2013; Wichadee, 2015), de las PTM así como la facilidad para conectarse o mantener el contacto con los docentes y los compañeros (Wichedee, 2015; Eskandari y Soleimani, 2016).

En relación a la claridad con la que se muestra la estructura organizativa de la asignatura en la PTM, las alumnas están más de acuerdo que los alumnos en qué ocurre con los contenidos; la metodología; la evaluación; además, consideran que se accede de forma fácil; prefieren aprender en asignaturas organizadas en temas secuenciados a través de la PTM; y que las actividades que se manejan a través de las plataformas de teleformación incitan a involucrarse en el propio aprendizaje.

Por último, apuntar desde aquí la necesidad expresada por los estudiantes de que los docentes estén más conectados a la realidad de lo que ocurre en la plataforma, dedicando más tiempo a la organización y desarrollo de la materia virtualizada (Wu y Hung, 2013).

\section{REFERENCIAS BIBLIOGRÁFICAS}

Alaminos, A. (2006). El muestreo en la investigación social. En A. Alaminos y J. L. Castejón, Elaboración, análisis $e$ interpretación de encuestas, cuestionarios y escalas de opinión (4667). Alcoy: Marfil.

Armijo de Vega, C., y McAnally-Salas, L. (2011). Finding Support in Moodle: A Face-to-Face Chemistry Course for Engineers. Online Submission, US-China Education Review, A1, 10-21.

Boza, A., y Conde, S. (2015). Web 2.0 en educación superior: formación, actitud, uso, impacto, dificultades y herramientas. Digital Educational Review, 48, 45-58. Recuperado de http://revistes.ub.edu
index.php/der/article/viewFile/14423/ pdf

Castaño, R., Jenaro, C., y Flores, N. (2012). Análisis DAFO de la utilidad de las plataformas de formación online para el entrenamiento en competencias de estudiantes universitarios. Edutec, 42. Recuperado de http://www.edutec.es revista/index.php/edutec-e/article/ view/346

Danciu, E., y Grosseck, G. (2011). Social aspects of web 2.0 technologies: teaching or teachers' challenges? Procedia Social and Behavioral Sciences, 15, 3768-3773. doi: 10.1016/j.sbspro.2011.04.371

De Pablos, J., Colás, M. P., y González, T. (2011). La enseñanza universitaria 
apoyada en plataformas virtuales. Cambios en las prácticas docentes: el caso de la Universidad de Sevilla. Estudios sobre Educación, 2O, 23-48. Recuperado de http://www.unav.edu/publicaciones/ revistas/index.php/estudios-sobreeducacion/article/viewFile/4462/3844

Emelyanova, N., y Voronina, E. (2014). Introducing a Learning Management System at a Russian University: Students' and Teachers' Perceptions. International Review of Research in Open and Distance Learning, 15(1), 272-289. Recuperado de http://www.irrodl.org/index.php/irrodl/ article/view/1701/2819

Erol, C. C. (2015). New approaches in art educations: Moodle learning and content magnagamente system based art education. Global Journal of Arts Education, 5(2), 67-71. doi: http://dx.doi. org/10.18844/gjae.v5i2.248

Eskandari, M., y Soleimani, H. (2016). The Effect of Collaborative Discovery Learning Using MOODLE on the Learning of Conditional Sentences by Iranian EFL Learners. Theory and Practice in Lanquaqe Studies, 6(1), 153-163. doi: http://dx.doi.org/10.17507/tpls.0601.20

Felpeto, A., Rey, R., Fernández-Vázquez, A., y Garrote, D. (2015). Uso de plataformas e-learning y alfabetización digital en formación profesional a distancia. Revista de Estudios e Investigación en Psicología y Educación, 13, 163-167. doi: http:/ dx.doi.org/10.17979/reipe.2015.0.13.490

González, M. (2007). Las TIC como factor de innovación y mejora de la calidad de la enseñanza. En J. Cabero (Coord.), Tecnología educativa (219-232). Madrid: McGraw-Hill.

Green, L. S., Inan, F. A., y Denton, B. (2012). Examination of factors impacting student satisfaction with a new learning management system. TOJET: The Turkish Online Journal of Educational Technology. 13(3), 189-197.
Guel, S., Pintor, M. M., y Gómez, M. (2016). Indicadores para la evaluación del nivel de satisfacción del uso de Blackboard. Campus Virtuales, 5(1), 36-47. Recuperado de http://www.uajournals. com/campusvirtuales/es/revistaes/ numeroactual.html?id=149

Hernández, R., Fernández, C., y Baptista, P. (2006). Metodología de la investigación. (6 ${ }^{\mathrm{a}}$ edición). México: McGraw Hill Interamericana.

Juhary, J. (2014). Perceived Usefulness and Ease of Use of the Learning Management System as a Learning Tool. International Education Studies, 7(8), 23-34.

Kopcha, T. (2010). A systems-based approach to technology integration using mentoring and communities of practice. Educational Technology Research and Development, 58, 175-190.

Ku, H.-Y., Tseng, H.-W., y Akarasriwo, C. (2013). Collaboration factors, teamwork satisfaction and student attitudes toward online collaborative learning. Computers inhuman Behavior, 29, 922-929.

Lai, A., y Savage, P. (2013). Learning Management Systems and Principles of Good Teaching: Instructor and Student Perspectives. Canadian Journal of Learning and Technology, 39(3) Recuperado de http://www.cjlt.ca/index. php/cjlt/article/view/699

Marín, V., y Cabero, J. (2015). Innovando en el aula universitaria a través de DIPRO 2.0. Revista Sophia, 11(2), 155168. Recuperado de http://revistas. ugca.edu.co/index.php/sophia/article/ view/348/521

Marín, V., Reche, E., y Maldonado, G. (2013). Ventajas e inconvenientes de la formación online. Revista Digital de Investigación en Docencia Universitaria, 7(1), 33-43. Recuperado de http://www3.upc.edu.pe/ html/o/documentos/ridu2013/articulo3riduu-33-43.pdf

Mateo, J. (2012). La investigación ex post-facto. En R. Bisquerra (Coord.), 
Metodología de la investigación educativa (195-230). Madrid: La Muralla. Maz, A., Bracho, R., Jiménez, N., y Adamuz, N. (2012). El foro en la plataforma Moodle: un recurso de la participación cooperativa para el aprendizaje de las matemáticas. EDMETIC, Revista de Educación Mediática y TIC, 1(2), 2943. Recuperado de http://www.uco.es/ ucopress/ojs/index.php/edmetic/article/ view $/ 2850 / 2738$

Mirabal, A. R., Gómez, M. G., y González, L. A. (2015). Uso de la plataforma Moodle como apoyo a la docencia presencial universitaria. EDMETIC, Revista de Educación Mediática y TIC, 4(1), 133155. Recuperado de http://www.uco.es/ ucopress/ojs/index.php/edmetic/article/ view/2903/2831

Morales, P. (2012). Estadística aplicada a las Ciencias Sociales. Tamaño necesario de la muestra: ¿cuántos sujetos necesitamos? Recuperado de http://www2.df.gob.mx/ virtual/evaluadf/docs/gral/taller2015/ SO202EAC.pdf

Pérez, R., García, J. L., Gil, J. A., y Galán, A. (2009). Estadística aplicada a la educación. Madrid: Pearson Educación y UNED.

Revuelta, F. I., y Pérez, L. (2009). Interactividad en los entornos de formación online. Barcelona: UOC.

Rivadulla, J. C. (2015). Concepciones de los estudiantes de Magisterio sobre Moodle. Revista de Estudios e Investigación en Psicología y Educación, 13, 68-72. Recuperado de http://revistas.udc.es index.php/reipe/article/view/328/ pdf 370

Rodríguez, M., y Rivadulla, J. C. (2015). La integración de plataformas de e-learning en la docencia universitaria: percepciones de un grupo de estudiantes sobre los usos de la plataforma Moodle. Revista Electrónica de Investigación y Docencia (REID), 14, 27-46. Recuperado de http:// revistaselectronicas.ujaen.es/index.php/ reid/article/view/2237

Sam, T. L. (2015). E-learning bencharking surrey: a case of University Utara Malysia. Universal Journal of Educational Research, 3(4), 269-276.

Srichanyachon, N. (2014). EFL learners' perceptions of using LMS. TOJET: The Turkish Online Journal of Educational Technology, 13(4), 30-35.

Tello, I., De Miguel, L., y López, M. D. (2012). Entornos personales de aprendizaje en el Espacio Europeo de Educación Superior. RIED. Revista Iberoamericana de Educación a Distancia, 1(2), 123142. Recuperado de http://ried.utpl. edu.ec/sites/default/files/file/archivo/ volumen\%2015 2/Entornospersonales. pdf

Wichadee, S. (2015). Factors Related to Faculty Members' Attitude and Adoption of a Learning Management System. TOJET: The Turkish Online Journal of Educational Technology, 14(4), 53-61.

Wu, Y.-C. J., y Huang, S. K. (2013). Making on-line logistics training sustainable through e-learning. Computer in Human Behavior, 29, 323-328. doi: 10.1016/j. chb.2012.07.027.

\section{PERFIL ACADÉMICO Y PROFESIONAL DEL AUTORES}

Verónica Marín-Díaz. Profesora Titular de Universidad de la Facultad de Ciencias de la Educación de la Universidad de Córdoba, ha sido Directora del Máster en Educación Inclusiva de la citada universidad en el período 2012-2015. Miembro del grupo de investigación e2i de la Universidad de Córdoba y del Grupo de Tecnología Educativa de la Universidad de Sevilla. Editora de la revista internacional 
EDMETIC, Revista de Educación Mediática y TIC. Sus líneas de investigación giran en torno a las tecnologías emergentes.

E-mail: vmarin@uco.es

Begoña E. Sampedro-Requena. Profesora acreditada a Ayudante Dra. y Contratada Dr. por la ANECA y la DEVA, docente de la Facultad de Ciencias de la Educación de la Universidad de Córdoba. Miembro del grupo de investigación e2i de la Universidad de Córdoba y del Grupo de Tecnología Educativa de la Universidad de Sevilla. Sus líneas de investigación giran en torno a las tecnologías emergentes. E-mail: bsampedro@uco.es

Esther Vega-Gea. Profesora acreditada a Ayudante Dr. por la DEVA, docente de la Facultad de Ciencias de la Educación de la Universidad de Córdoba. Miembro del grupo de investigación Laboratorio de Estudios sobre Convivencia y Prevención de la violencia de la Universidad de Córdoba Sus líneas de investigación giran en torno a las tecnologías emergentes.

E-mail: esther.vega@uco.es

\section{DIRECCIÓN DE LAS AUTORAS}

Facultad de Ciencias de la Educación

Universidad de Córdoba

Facultad de Ciencias de la Educación

Avda. San Alberto Magno s/n

14004-Córdoba (España)

Fecha de recepción del artículo: 08/05/2016

Fecha de aceptación del artículo: 12/07/2016

\section{Como citar este artículo:}

Marín Díaz, V., Sampedro Requena, B. E., y Vega Gea, E. (2017). Percepciones de los estudiantes universitarios sobre las plataformas de formación. Estudio de caso. RIED. Revista Iberoamericana de Educación a Distancia, 20(1), pp. 283-303. doi: http://dx.doi.org/10.5944/ried.20.1.16518 